\title{
Faired MISO B-Spline Fuzzy Systems and Its Applications
}

\author{
Tan Yanhua ${ }^{1,2}$ and Li Hongxing ${ }^{1}$ \\ ${ }^{1}$ Faculty of Electronic Information and Electrical Engineering, Dalian University of Technology, Dalian, Liaoning 116024, China \\ ${ }^{2}$ School of Sciences, Hebei University of Technology, Tianjin 300130, China
}

Correspondence should be addressed to Tan Yanhua; tanyh@hebut.edu.cn

Received 11 December 2012; Accepted 7 February 2013

Academic Editor: Wudhichai Assawinchaichote

Copyright (C) 2013 T. Yanhua and L. Hongxing. This is an open access article distributed under the Creative Commons Attribution License, which permits unrestricted use, distribution, and reproduction in any medium, provided the original work is properly cited.

\begin{abstract}
We construct two classes of faired MISO B-spline fuzzy systems using the fairing method in computer-aided geometric design (CAGD) for reducing adverse effects of the inexact data. Towards this goal, we generalize the faring method to high-dimension cases so that the faring method only for SISO and DISO B-spline fuzzy systems is extended to fair the MISO ones. Then the problem to construct a faired MISO B-spline fuzzy systems is transformed into solving an optimization problem with a strictly convex quadratic objective function and the unique optimal solution vector is taken as linear combination coefficients of the basis functions for a certain B-spline fuzzy system to obtain a faired MISO B-spline fuzzy system. Furthermore, we design variable universe adaptive fuzzy controllers by B-spline fuzzy systems and faired B-spline fuzzy systems to stabilize the double inverted pendulum. The simulation results show that the controllers by faired B-spline fuzzy systems perform better than those by B-spline fuzzy systems, especially when the data for fuzzy systems are inexact.
\end{abstract}

\section{Introduction}

Since Zadeh introduced fuzzy theory in 1965, fuzzy systems have been utilized successfully in many areas, such as fuzzy control, classification, expert systems, and others. It is known that a fuzzy system is usually established by input-output data (I/O data) which can be obtained by experiments, expert knowledge, or observation records. However, the accuracy of these I/O data may be affected by hardware/software limitations, unavoidable round off, truncation error of a system, and some uncertainties [1]. This means that we cannot establish fuzzy systems on the exact I/O data. Therefore, it is important to construct appropriate fuzzy systems when the I/O data is inexact. Reference [2] gives the upper bounds of the output errors of two kinds of fuzzy systems affected by the perturbation of I/O data. However, the problem of performance improvement for fuzzy systems is seldom considered in the case of the inexact $\mathrm{I} / \mathrm{O}$ data.

Fuzzy systems can be constructed by splines [3-6] because the design of a fuzzy system can be regarded as a function approximation problem [7-10] and spline functions have many nice structural properties and excellent approximation powers [11]. By investigating the relation between fuzzy systems and splines, we proposed two classes of B-spline fuzzy systems (B-FSs) [12, 13], which are linear combination of B-spline basis functions and rational B-spline basis functions, respectively. Therefore, the single input single output (SISO) and double input single output (DISO) of these two classes of B-FSs can be regarded as curves and surfaces in CAGD. As curves and surfaces in CAGD, fairness is necessary. Though fairness is the property about geometric shapes, a fair curve can seek through the digitizing errors in its design process [14]. Hence, it is necessary to reduce adverse effects of the inexact I/O data on a fair fuzzy system. Since BFSs can be regarded as curves and surfaces in CAGD, we can fair them and obtain good performance.

In this paper, we construct two classes of faired B-spline fuzzy systems (faired B-FSs) to reduce adverse effects of the inexact I/O data on fuzzy systems as well as improve their performance. For faring these two classes of B-FSs, the energy extremum principle (energy method) based faring method in CAGD is utilized for its overall modification nature. However, we note that the energy method in CAGD is only used to fair curves and surfaces, which means it can only fair the SISO and DISO B-FSs. So, we propose a regularization term taken as the energy function of the MISO B-FS. By using 
this energy function, the energy method in CAGD, which can only be applied to fairing SISO and DISO B-FSs, is extended to fair the MISO ones. Therefore, based on the above preparations, the problem to construct a faired MISO B-FS is transformed into solving an optimization problem with a strictly convex quadratic objective function. In our proposed method, the faired MISO B-FS is available by taking the unique optimal solution vector as linear combination coefficients of the corresponding MISO B-FS.

As we all know, fuzzy controllers are a type of closed-loop fuzzy systems, while adaptive fuzzy controllers are closedloop fuzzy systems with adaptive or training algorithms [1519]. Especially, Professor Li advances the variable universe method [20-22], and this method succeeded in the experiment of controlling the simulation model and physical model of quadruple inverted pendulum with variable universe fuzzy controllers in 2001 [23] and 2002. In order to verify the ability of the faired B-FSs, we design variable universe adaptive fuzzy controllers by B-FSs and faired B-FSs to stabilize the double inverted pendulum. The simulation results show that the controllers by faired B-FSs perform better than those by B-FSs, especially when the I/O data for fuzzy systems are inexact.

The paper is organized as follows. Section 2 provides some preliminaries. The faired MISO B-FSs are constructed in Section 3. In Section 4, the variable universe adaptive fuzzy controllers by B-FSs and faired B-FSs are designed to demonstrate their ability. The final section contains some conclusions and prospects of our research.

\section{Preliminaries}

In this section, we will introduce the definition of B-spline basis functions, the Frobenius norm, and briefly review the two classes of MISO B-FSs in $[12,13]$.

Definition 1 (B-spline basis functions [24]). Let $U=$ $\left\{u_{0}, \ldots, u_{m}\right\}$ be a nondecreasing sequence of real numbers, that is, $u_{i} \leq u_{i+1}, i=0, \ldots, m-1$. The $u_{i}$ is called knots, and $U$ is the knot vector. The $i$ th B-spline basis function of $p$-degree (order $p+1)$, denoted by $N_{i, p}(u)$, is defined as

$$
\begin{aligned}
N_{i, 0}(u) & = \begin{cases}1, & \text { if } u_{i} \leq u<u_{i+1}, \\
0, & \text { otherwise, }\end{cases} \\
N_{i, p}(u)= & \frac{u-u_{i}}{u_{i+p}-u_{i}} N_{i, p-1}(u) \\
& +\frac{u_{i+p+1}-u}{u_{i+p+1}-u_{i+1}} N_{i+1, p-1}(u) .
\end{aligned}
$$

Definition 2 (Frobenius norm [25]). The Frobenius norm on $\mathbb{R}^{n \times n}$ is defined as

$$
\|A\|_{F}=\left(\sum_{i=1}^{m} \sum_{j=1}^{n}\left|a_{i j}\right|^{2}\right)^{1 / 2}, \quad A=\left(a_{i j}\right) \in \mathbb{R}^{n \times n} .
$$

Obviously,

$$
\|A\|_{F}=\left(\operatorname{Tr}\left(A^{T} A\right)\right)^{1 / 2} .
$$

In fuzzy inference, to acquire a group of inference rules and to acquire a group of $\mathrm{I} / \mathrm{O}$ data are the same thing [10]. In this paper, we always denote the I/O data for MISO fuzzy systems as

$$
\left\{\left(u_{1, j_{1}}, u_{2, j_{2}}, \ldots, u_{n, j_{n}}, v_{j_{1} j_{2} \cdots j_{n}}\right) \mid j_{i}=0,1, \ldots, p_{i}, i=1, \ldots, n\right\},
$$

where $u_{i, 0}=a_{i}, u_{i, p i}=b_{i}$, and $u_{i, 0}<u_{i, 1}<\cdots<u_{i, p_{i}}, i=$ $1, \ldots, n$.

Let $A_{i, j_{i}}(x)=N_{j_{i}-2,3}(x)$, where $N_{j_{i}-2,3}(x)$ is the cubic Bspline basis function with knots $u_{i, j_{i}-2}, u_{i, j_{i}-1}, u_{i, j_{i}}, u_{i, j_{i}+1}$, and $u_{i, j_{i}+2}$. The two classes of MISO B-FSs we proposed in [12,13] are listed below.

(1) The MISO first class of B-spline fuzzy system (1-B-FS) is

$$
\begin{aligned}
& F_{1}\left(x_{1}, x_{2}, \ldots, x_{n}\right) \\
& =\sum_{j_{1}=-1}^{p_{1}+1} \sum_{j_{2}=-1}^{p_{2}+1} \cdots \sum_{j_{n}=-1}^{p_{n}+1} A_{1, j_{1}}\left(x_{1}\right) A_{2, j_{2}}\left(x_{2}\right) \cdots A_{n, j_{n}}\left(x_{n}\right) \omega_{j_{1} j_{2} \cdots j_{n}},
\end{aligned}
$$

where $\omega_{j_{1} j_{2} \ldots j_{n}}, j_{i}=-1,0, \ldots, p_{i}+1, i=1, \ldots, n$ are obtained by solving equations

$$
\begin{gathered}
F_{1}\left(u_{1, j_{1}}, u_{2, j_{2}}, \ldots, u_{n, j_{n}}\right)=v_{j_{1} j_{2} \ldots j_{n}}, \\
j_{i}=-1,0, \ldots, p_{i}+1, i=1, \ldots, n,
\end{gathered}
$$

after extrapolating some points.

(2) The MISO second class of B-spline fuzzy system (2-BFS) is

$$
\begin{aligned}
& F_{2}\left(x_{1}, x_{2}, \ldots, x_{n}\right) \\
& =\sum_{j_{1}=0}^{p_{1}} \sum_{j_{2}=0}^{p_{2}} \cdots \\
& \sum_{j_{n}=0}^{p_{n}} \frac{A_{1, j_{1}}\left(x_{1}\right) A_{2, j_{2}}\left(x_{2}\right) \cdots A_{n, j_{n}}\left(x_{n}\right)}{\sum_{l_{1}=0}^{p_{1}} \sum_{l_{2}=0}^{p_{2}} \cdots \sum_{l_{n}=0}^{p_{n}} A_{1, l_{1}}\left(x_{1}\right) A_{2, l_{2}}\left(x_{2}\right) \cdots A_{n, l_{n}}\left(x_{n}\right)} \\
& \quad \times v_{j_{1} j_{2} \cdots j_{n}} .
\end{aligned}
$$

\section{The Faired MISO B-FSs}

In this section, two classes of the faired MISO B-FSs are constructed. In order to fair them together, we write them in the unification SISO form. By analyzing the energy 
functions of SISO and DISO B-FS, the energy function of MISO B-FS is proposed, which is a regularization term essentially. Consequently, the energy method is suitable for fairing the MISO B-FSs. Then, we transform the problem to construct a faired MISO B-FS into solving an unconstrained optimization problem. Based on the unification SISO form, the objective function of the unconstrained optimization problem can be reduced to a quadratic function which is turned to a strictly convex quadratic function via using a proper weight. Therefore, the unique optimal solution is available through solving linear equations of the first-order optimality condition. Consequently, the faired MISO B-FS is obtained by taking the unique optimal solution vector as linear combination coefficients of the corresponding B-FS. In the following, we will describe the above procedure in details.

3.1. The Unification SISO Form of Two Classes of B-FSs. The uniform form of (5) and (7) is,

$$
F(\mathbf{x})=\sum_{k=1}^{N} C_{k}(\mathbf{x}) \alpha_{k}=\alpha^{T} \mathbf{C}(\mathbf{x}),
$$

where $\mathbf{x}=\left(x_{1}, x_{2}, \ldots, x_{n}\right), \alpha=\left(\alpha_{1}, \alpha_{2}, \ldots, \alpha_{N}\right)^{T}$, and $\mathbf{C}(\mathbf{x})=$ $\left(C_{1}(\mathbf{x}), C_{2}(\mathbf{x}), \ldots, C_{N}(\mathbf{x})\right)^{T}$.

If we have

$$
\begin{gathered}
C_{k}(\mathbf{x})=A_{1, j_{1}}\left(x_{1}\right) A_{2, j_{2}}\left(x_{2}\right) \cdots A_{n, j_{n}}\left(x_{n}\right), \\
\alpha_{k}=\omega_{j_{1} j_{2} \cdots j_{n}}, \\
k=\left(j_{1}+2\right)+\left(j_{2}+1\right)\left(p_{1}+3\right)+\cdots \\
\quad+\left(j_{n}+1\right)\left(p_{1}+3\right) \cdots\left(p_{n-1}+3\right), \\
j_{i}=-1,0, \ldots, p_{i}+1, \quad i=1,2, \ldots, n, \\
N=\left(p_{1}+3\right)\left(p_{2}+3\right) \cdots\left(p_{n}+3\right),
\end{gathered}
$$

then (8) will turn to the 1-B-FS (5).

While, if we have

$$
\begin{aligned}
& C_{k}(\mathbf{x}) \\
& =\frac{A_{1, j_{1}}\left(x_{1}\right) A_{2, j_{2}}\left(x_{2}\right) \cdots A_{n, j_{n}}\left(x_{n}\right)}{\sum_{l_{1}=0}^{p_{1}} \sum_{l_{2}=0}^{p_{2}} \cdots \sum_{l_{n}=0}^{p_{n}} A_{1, l_{1}}\left(x_{1}\right) A_{2, l_{2}}\left(x_{2}\right) \cdots A_{n, l_{n}}\left(x_{n}\right)}, \\
& \alpha_{k}=v_{j_{1} j_{2} \cdots j_{n}}, \\
& k=\left(j_{1}+1\right)+j_{2}\left(p_{1}+1\right)+\cdots \\
& \quad+j_{n}\left(p_{1}+1\right) \cdots\left(p_{n-1}+1\right), \\
& j_{i}=0,1, \ldots, p_{i}, \quad i=1,2, \ldots, n, \\
& N=\left(p_{1}+1\right)\left(p_{2}+1\right) \cdots\left(p_{n}+1\right),
\end{aligned}
$$

then (8) is exactly the 2-B-FS (7). Consequently, (8) is named as the unification SISO form of the two classes of B-FSs.
3.2. Generalizing the Energy Method to High-Dimension Cases. The objects studied in CAGD are curves and surfaces which are parametric equations with single parameter and double parameters, respectively. The problem of fairing a curve (surface) by the energy method can be transformed into the following optimal one [26]:

$$
\min _{V} E(V)+w D(V)
$$

where the variable $V$ is the vector of control points, $E(V)$ is the energy function, $D(V)$ is the difference between the faired data points and the original ones, and $w$ is the weight which is assigned in advance. In CAGD, an approximated or simplified strain energy is used as energy function $E(V)$ [26]. For SISO and DISO B-FS, which can be regarded as curve and surface in CAGD, the energy functions can be written as

$$
\begin{gathered}
E_{1}=\int_{a}^{b}\left(F^{\prime \prime}(x)\right)^{2} \mathrm{~d} x \\
E_{2}=\iint_{D}\left(\left(\frac{\partial^{2} F}{\partial x_{1}^{2}}\right)^{2}+2\left(\frac{\partial^{2} F}{\partial x_{1} \partial x_{2}}\right)^{2}+\left(\frac{\partial^{2} F}{\partial x_{2}^{2}}\right)^{2}\right) \mathrm{d} x_{1} \mathrm{~d} x_{2} .
\end{gathered}
$$

By (2) and (3), the integrand of $E$ in (12) or (13) is the square of the Frobenius norm of $\nabla^{2} F(\mathbf{x})$ (the Hessian matrix of $F(\mathbf{x})$ ). That is,

$$
\begin{aligned}
E & =\int_{D}\left\|\nabla^{2} F(\mathbf{x})\right\|_{F}^{2} \mathrm{~d} \mathbf{x} \\
& =\int_{D} \operatorname{Tr}\left[\left(\nabla^{2} F(\mathbf{x})\right)^{T} \nabla^{2} F(\mathbf{x})\right] \mathrm{d} \mathbf{x} .
\end{aligned}
$$

When the number of input variable is more than 2 , it is difficult to get an energy function with specific geometric meaning or physical meaning. In order to fair the B-FSs with more than 2 input variables, we have to generalize the energy method to the high-dimension cases. In fact, the energy method is a kind of regularization method with the energy function as its regularization term. Especially, when the observation data is inexact, the regularization method can identify a meaningful and stable solution [27]. This coincides with our motive to fair. It is noted that (14) can be viewed as a regularization term. Consequently, we call the regularization method with (14) as its regularization term the generalized energy method. It is known that (14) is the energy function for MIMO B-FS. Therefore, we generalize the energy method to the high-dimension cases.

3.3. The Faired MISO B-FSs. In general, the curves (surfaces) in CAGD are referred to the parametric B-spline curves (surfaces), while the B-FSs are the B-spline functions. When the parametric B-spline curves (surfaces) degenerate into Bspline functions, the control points will be the coefficients of B-spline basis functions. Write the original B-FS and the faired B-FS as

$$
\begin{aligned}
& F_{0}(\mathbf{x})=\alpha_{0}^{T} \mathbf{C}(\mathbf{x}), \\
& F(\mathbf{x})=\alpha^{T} \mathbf{C}(\mathbf{x}),
\end{aligned}
$$


respectively. Then, by (11), we can transform the problem to construct a faired MISO B-FS into solving the following unconstrained optimization problem as

$$
\min _{\alpha \in R^{N}} H(\alpha) \triangleq E+w\left(\alpha^{T}-\alpha_{0}^{T}\right)\left(\alpha-\alpha_{0}\right),
$$

where $w$, the positive weight, is assigned in advance, and $E$ is defined as in (14).

From the following two extreme cases, we can recognize the concrete significance of $w$.

(1) When $w \rightarrow \infty$, since $H=\min$, we have

$$
\alpha=\alpha_{0}
$$

Thus the faired B-FS $F(\mathbf{x})$ turns to the original BFS $F_{0}(\mathbf{x})$, which implies that the B-FS $F_{0}(\mathbf{x})$ is not modified after the fairing process.

(2) When $w=0$, we immediately get that $E=0$ from $H=\min$. Therefore, the B-FS $F(\mathbf{x})$ turns to be the fairest one in this case.

For the cases in between, when $w$ is set to be a small number, $E$ becomes small and the fuzzy system (16) is fair at the cost of much difference between $F(\mathbf{x})$ and $F_{0}(\mathbf{x})$ as well as much difference between $F\left(\mathbf{u}_{s}\right)$ and $v_{s}$. On the other hand, the larger $w$ is, the less the difference is between $F(\mathbf{x})$ and $F_{0}(\mathbf{x})$. Thus there is less difference between $F\left(\mathbf{u}_{s}\right)$ and $v_{s}$. And the fairness of the fuzzy system (16) may be poor. So when the $\mathrm{I} / \mathrm{O}$ data is inexact, a smaller $w$ is needed to make a larger difference between $F(\mathbf{x})$ and $F_{0}(\mathbf{x})$, and then the original BFS $F_{0}(\mathbf{x})$ may be improved. On the contrary, a larger $w$ might be appropriate.

Let $D=\left[a_{1}, b_{1}\right] \times\left[a_{2}, b_{2}\right] \times \cdots \times\left[a_{n}, b_{n}\right]$, and substitute (8) into (14), we have,

$$
\begin{aligned}
E & =\int_{D} \sum_{i=1}^{n} \sum_{j=1}^{n}\left(\frac{\partial^{2} F}{\partial x_{i} \partial x_{j}}\right)^{2} \mathrm{~d} \mathbf{x} \\
& =\alpha^{T}\left(\int_{D} \sum_{i=1}^{n} \sum_{j=1}^{n} \frac{\partial \mathbf{C}}{\partial x_{i} \partial x_{j}}\left(\frac{\partial \mathbf{C}}{\partial x_{i} \partial x_{j}}\right)^{T} \mathrm{~d} \mathbf{x}\right) \alpha .
\end{aligned}
$$
is

Then, the objective function of the optimal problem (17)

$$
H(\alpha)=\alpha^{T}(A+w I) \alpha-2 w \alpha^{T} \alpha_{0}+w \alpha_{0}^{T} \alpha_{0}
$$

where

$$
A=\int_{D} \sum_{i=1}^{n} \sum_{j=1}^{n} \frac{\partial \mathbf{C}}{\partial x_{i} \partial x_{j}}\left(\frac{\partial \mathbf{C}}{\partial x_{i} \partial x_{j}}\right)^{T} \mathrm{~d} \mathbf{x} .
$$

From (20), we know that $H(\alpha)$ is a quadratic function. Moreover, by (21), $A+w I$ is a symmetric matrix. Obviously, there always exists weight $w$ to make $A+w I$ a symmetric positive definite matrix. Thus, $H(\alpha)$ becomes a strictly convex quadratic function. Consequently, through solving the linear

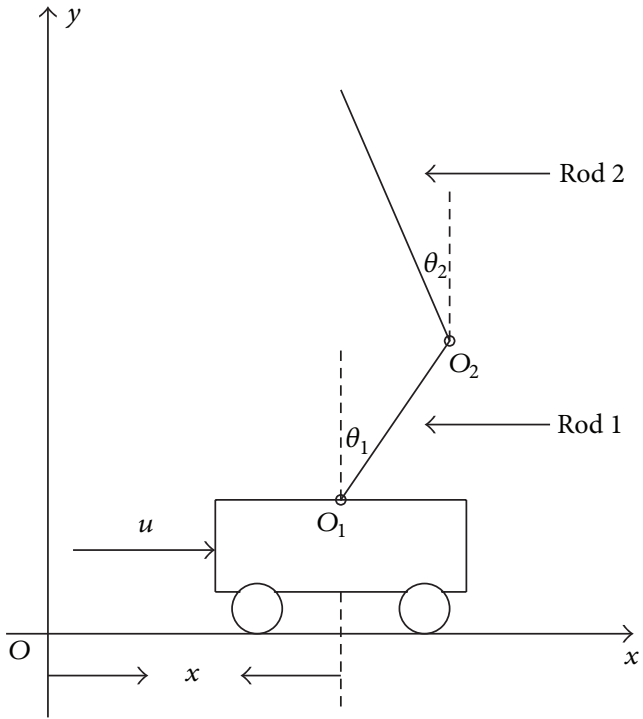

FIGURE 1: A simplified model of the double inverted pendulum.

equations of the first-order optimality condition as shown in the following:

$$
(A+w I) \alpha^{*}=w \alpha_{0}
$$

we can get the unique optimal solution $\alpha^{*}$ of (17). Let $\alpha^{*}$ serve as the linear combination coefficients of (16), the faired MISO $\mathrm{B}-\mathrm{FS}$ is available.

Remark 3. Since it is convenient to deal with a uniform cubic B-spline, when the knots of the B-spline basis functions of a B-FS are arbitrary (non-uniform), we can approximate to this B-FS by one fuzzy system with uniform cubic B-spline basis functions to fair it.

\section{Algorithm. Constructing a Faired MISO B-FS.}

Step 1. Extract I/O data (4) from the fuzzy inference rules;

Step 2. Given the initial weight $w$;

Step 3. Calculate $A$ by (21);

Step 4. If $A+w I$ is positive definite, the optimal solution is available by solving linear equations (22). Otherwise, let $w=$ $2 w$, go to Step 4;

Step 5. Evaluate the obtained faired B-FS, $F(\mathbf{x})$, if it works, end, if not, tuning the weight $w$, go to Step 4 .

\section{Simulation Results}

In this section, we design variable universe adaptive fuzzy controllers by B-FSs (hereinafter abbreviated as B-FCs) and faired B-FSs (hereinafter abbreviated as faired B-FCs) to stabilize the double inverted pendulum. Moreover, the control effect between them is compared. As the analysis 
TABLE 1: Control rules for double inverted pendulum.

\begin{tabular}{cccccccc}
\hline ec & & & & $e$ & $A_{5}$ & $A_{6}$ \\
& $A_{1}$ & $A_{2}$ & $A_{3}$ & $A_{4}$ & -0.1667 & 0 \\
\hline$B_{1}$ & -0.8333 & -0.8333 & -0.6333 & -0.5 & -0.3333 & 0.1667 \\
$B_{2}$ & -0.8333 & -0.6333 & -0.5 & -0.3333 & -0.1667 & 0 & 0.1667 \\
$B_{3}$ & -0.6333 & -0.5 & -0.3333 & -0.1667 & 0 & 0.1633 \\
$B_{4}$ & -0.5 & -0.3333 & -0.1667 & 0 & 0.3333 \\
$B_{5}$ & -0.3333 & -0.1667 & 0 & 0.1667 & 0.3333 & 0.5 \\
$B_{6}$ & -0.1667 & 0 & 0.1667 & 0.3333 & 0.5 & 0.6333 \\
$B_{7}$ & 0 & 0.1667 & 0.3333 & 0.5 & 0.6333 & 0.8333 \\
\hline
\end{tabular}
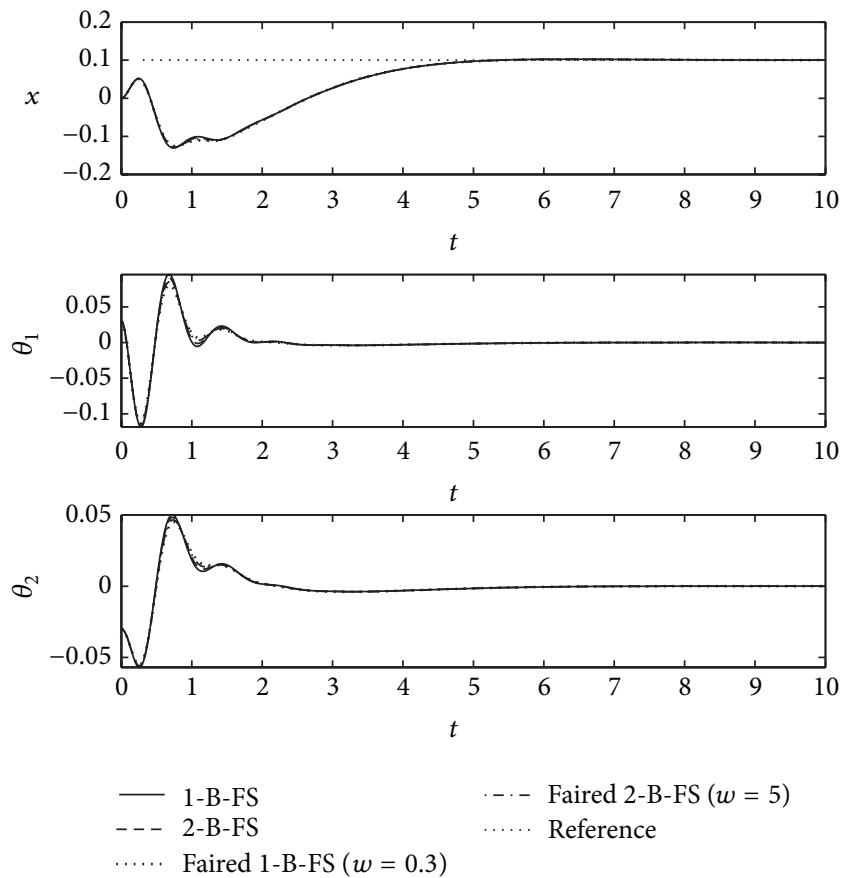

Figure 2: Response of controllers by 1-B-FS, 2-B-FS, faired 1-B-FS, and faired 2-B-FS with I/O data DISODS.

in Section 3.3, the weight $w$ can affect the fairness and difference of the faired B-FSs. Further, it may affect the control effect of the faired B-FCs, too. Therefore, the control effect incited by different weights is demonstrated in the following simulations.

The double inverted pendulum is mainly made up of a cart, two rods which are freely linked together. The case where they are put in a coordinate system is shown in Figure 1.

Let the clockwise angle and moment in Figure 1 be in positive direction. And we assume that $u$ is the outer force of system, $x$ the displacement of the cart, $\theta_{i}$ the angle between rod $i$ and vertical direction, $O_{i}$ and $G_{i}$ the linking point and centroid of rod $i, m_{0}$ the mass of the cart, $m_{i}$ the mass of rod $i, J_{i}$ the moment of inertia of $\operatorname{rod} i$ around $O_{i}, l_{i}$ the distance from $O_{i}$ to $G_{i}, L_{i}$ the length of $\operatorname{rod} i, f_{0}$ the fricative coefficient between the cart and its orbit, and $f_{i}$ the fricative coefficient of rod $i$ around $O_{i}(i=1,2)$. Then the differential equations to describe the locomotion of the double inverted pendulum are

$$
H_{1}(z) \dot{z}=H_{2}(z) z+H_{3}(z)+H_{0} u
$$

where $z=\left(x, \theta_{1}, \theta_{2}, \dot{x}, \dot{\theta}_{1}, \dot{\theta}_{2}\right)^{T}, a_{1}=m_{1} l_{1}+m_{2} L_{1}, a_{2}=$ $m_{2} l_{2}, b_{1}=J_{1}+m_{2} L_{1}^{2}, b_{2}=J_{2}$, and

$$
\begin{aligned}
& H_{1}(z)= \\
& \left(\begin{array}{cccccc}
1 & 0 & 0 & 0 & 0 & 0 \\
0 & 1 & 0 & 0 & 0 & 0 \\
0 & 0 & 1 & 0 & 0 & 0 \\
0 & 0 & 0 & 1 & 0 & 0 \\
0 & 0 & 0 & a_{1} \cos \theta_{1} & b_{1} & a_{2} L_{1} \cos \left(\theta_{2}-\theta_{1}\right) \\
0 & 0 & 0 & a_{2} \cos \theta_{2} & a_{2} L_{1} \cos \left(\theta_{2}-\theta_{1}\right) & b_{2}
\end{array}\right), \\
& H_{2}(z)= \\
& \left(\begin{array}{cccccc}
0 & 0 & 0 & 1 & 0 & 0 \\
0 & 0 & 0 & 0 & 1 & 0 \\
0 & 0 & 0 & 0 & 0 & 0 \\
0 & 0 & 0 & 0 & 0 & a_{2} L_{1} \dot{\theta}_{2} \sin \left(\theta_{2}-\theta_{1}\right)+f_{2} \\
0 & 0 & 0 & 0 & -f_{1}-f_{2} & -f_{2} \\
0 & 0 & 0 & 0 & -a_{2} L_{1} \dot{\theta}_{1} \sin \left(\theta_{2}-\theta_{1}\right)+f_{2} &
\end{array}\right.
\end{aligned}
$$

$H_{3}(z)=\left(0,0,0,0, a_{1} g \sin \theta_{1}, a_{2} g \sin \theta_{2}\right)^{T}$, and $H_{0}=$ $(0,0,0,1,0,0)^{T}$.

For the control system of the double inverted pendulum, our control aim is to make the angles $\theta_{1}, \theta_{2}$, respectively, converge to 0 and, at the same time, drive the cart to the point $x_{d}$ which is pointed out by us in advance. In this simulation experiment, the parameters in the double inverted pendulum are taken as $m_{1}=0.373, m_{2}=0.088$ (unit: $\mathrm{kg}$ ), $L_{1}=0.397$, $l_{1}=0.31815, L_{2}=0.345, l_{2}=0.15205$ (unit: $\mathrm{m}$ ), $J_{1}=$ $0.044048, J_{2}=0.00297947$ (unit: $\mathrm{kg} \cdot \mathrm{m}^{2}$ ), $f_{1}=0, f_{2}=0$ (unit: $\mathrm{N} \cdot \mathrm{s} \cdot \mathrm{m}$ ), and $g=9.81\left(\right.$ unit: $\mathrm{m} / \mathrm{s}^{2}$ ).

The variable universe adaptive fuzzy controller for the double inverted pendulum is designed by the method in [23]. Firstly, we linearize (23) at the equilibrium point $z=(0,0,0,0,0,0)$. Then, let $Q=\operatorname{diag}([1,1,1,1,1,1]), R=0.1$, and solve LQR by Matlab. And then we can get a state feedback matrix $k=(3.1623,-154.66,192.58,6.3854,-6.7368,26.806)$ and 
TABLE 2: Performance index of control systems by 1-B-FS, faired 1-B-FSs with I/O data DISODS.

\begin{tabular}{|c|c|c|c|c|c|}
\hline \multirow[t]{3}{*}{ State } & \multirow{2}{*}{$\begin{array}{c}\text { Index } \\
w\end{array}$} & \multirow[t]{2}{*}{ 1-B-FS } & \multicolumn{3}{|c|}{ Faired 1-B-FS } \\
\hline & & & 0.0002 & 0.3 & 2 \\
\hline & Diff & 0 & $4.65 \%$ & $1.19 \%$ & $0.953 \%$ \\
\hline$x$ & & 65.6 & 5.10 & 1.93 & 23.8 \\
\hline$\theta_{1}$ & Steady-state error $\left(10^{-9}\right)$ & 150 & 16.4 & 0.461 & 52.7 \\
\hline$\theta_{2}$ & & 33.0 & 9.24 & 3.65 & 13.5 \\
\hline$x$ & & 0.231 & 0.230 & 0.226 & 0.227 \\
\hline$\theta_{1}$ & Maximum overshoot & 0.118 & 0.111 & 0.113 & 0.115 \\
\hline$\theta_{2}$ & & 0.0570 & 0.0561 & 0.0554 & 0.0560 \\
\hline$x$ & & 3.36 & 3.44 & 3.33 & 3.34 \\
\hline$\theta_{1}$ & Settling & 0.822 & 0.826 & 0.820 & 0.839 \\
\hline$\theta_{2}$ & & 0.713 & 0.319 & 0.313 & 0.315 \\
\hline & $u^{2} \mathrm{~d} t$ & 6.27 & 4.94 & 5.27 & 5.61 \\
\hline
\end{tabular}

TABLE 3: Performance index of control systems by 2-B-FS, faired 2-B-FSs with I/O data DISODS.

\begin{tabular}{|c|c|c|c|c|c|}
\hline \multirow[t]{3}{*}{ State } & \multirow{2}{*}{$\begin{array}{c}\text { Index } \\
w\end{array}$} & \multirow[t]{2}{*}{ 2-B-FS } & \multicolumn{3}{|c|}{ Faired 2-B-FS } \\
\hline & & & 3 & 5 & 10 \\
\hline & Diff & $0.332 \%$ & $1.20 \%$ & $0.853 \%$ & $0.610 \%$ \\
\hline$x$ & \multirow{3}{*}{ Steady-state error $\left(10^{-9}\right)$} & 13.4 & 2.45 & 3.66 & 0.587 \\
\hline$\theta_{1}$ & & 14.4 & 2.45 & 6.18 & 0.973 \\
\hline$\theta_{2}$ & & 17.5 & 3.31 & 3.34 & 0.549 \\
\hline$x$ & \multirow{3}{*}{ Maximum overshoot } & 0.229 & 0.228 & 0.227 & 0.227 \\
\hline$\theta_{1}$ & & 0.117 & 0.116 & 0.115 & 0.114 \\
\hline$\theta_{2}$ & & 0.0565 & 0.0563 & 0.0559 & 0.0557 \\
\hline$x$ & \multirow{3}{*}{ Settling time } & 3.35 & 3.35 & 3.34 & 3.35 \\
\hline$\theta_{1}$ & & 0.823 & 0.825 & 0.802 & 0.812 \\
\hline$\theta_{2}$ & & 0.31 & 0.308 & 0.315 & 0.314 \\
\hline & $u^{2} \mathrm{~d} t$ & 5.90 & 5.80 & 5.55 & 5.44 \\
\hline
\end{tabular}

$\|k\|_{2}=248.64$. Let $k$ be the coefficient vector to reduce the dimensions and

$$
\begin{aligned}
& e \triangleq \frac{k(1) z(1)+k(2) z(2)+k(3) z(3)}{\|k\|_{2}}, \\
& e c \triangleq \frac{k(4) z(4)+k(5) z(5)+k(6) z(6)}{\|k\|_{2}}
\end{aligned}
$$

be a kind of integrated error and integrated error change rate. The variable universe adaptive fuzzy controller is

$$
\begin{aligned}
u= & \|k\|_{2}\left(\int_{0}^{t} 5(e+e c) F\left(\frac{e}{\alpha(e)}, \frac{e c}{\alpha(e c)}\right)\|k\|_{2} d t+1\right) \\
& \times F\left(\frac{e}{\alpha(e)}, \frac{e c}{\alpha(e c)}\right),
\end{aligned}
$$

where $F(\cdot)$ is a fuzzy system of two input variables.

The universe of $e(t)$ and $e c(t)$ are both taken as $[-1,1]$. The fuzzy partition of $e(t)$ is $A_{1}=\mathrm{NB}, A_{2}=\mathrm{NM}, A_{3}=\mathrm{NS}$, $A_{4}=\mathrm{ZO}, A_{5}=\mathrm{PS}, A_{6}=\mathrm{PM}, A_{7}=\mathrm{PB}$, and the fuzzy partition of $e c(t)$ is $B_{1}=\mathrm{NB}, B_{2}=\mathrm{NM}, B_{3}=\mathrm{NS}, B_{4}=\mathrm{ZO}$, $B_{5}=\mathrm{PS}, B_{6}=\mathrm{PM}, B_{7}=\mathrm{PB}$. The control rules are given in Table 1 [23].
So the relatively exact $\mathrm{I} / \mathrm{O}$ data for $F(\cdot)$ is that

$$
\text { DISODS } \triangleq\left\{\left(x_{i}, y_{j}, z_{i j}\right) \mid i=1,2, \ldots, 7, j=1,2, \ldots, 7\right\},
$$

where $\left(x_{1}, x_{2}, \ldots, x_{7}\right)=(-1,-2 / 3,-1 / 3,0,1 / 3,2 / 3,1)$, $\left(y_{1}, y_{2}, \ldots, y_{7}\right)=(-1,-2 / 3,-1 / 3,0,1 / 3,2 / 3,1)$, and matrix $\left(z_{i j}\right)_{7 \times 7}$ is formed by the data in Table 1 .

When the I/O data is inexact, we consider the data with noise only which is obtained by adding the Gaussian white noise. In this simulation, the inexact $\mathrm{I} / \mathrm{O}$ data is obtained by adding the Gaussian white noise with mean 0 and variance $(0.001)^{2}$ to I/O data DISODS, and the obtained inexact I/O data is denoted as DISODS ${ }^{1}$.

Let $\alpha(e)=1, \alpha(e c)=1, z_{0}=(0,0.03,-0.03,0,0,0)^{T}$, and $x_{d}=0.1$. Figures 2 and 3 show the control effect of B-FCs and faired B-FCs respectively, where Figure 3 is the average result of 100 independent runs. Obviously,

(1) when the I/O data is DISODS, the control effect of the B-FCs is nearly as good as that of the faired ones (Figure 2);

(2) when the I/O data is DISODS ${ }^{1}$, the faired B-FCs outperform that of the B-FCs (Figure 3). 
TABLE 4: Performance index of control systems by 1-B-FS, faired 1-B-FSs with I/O data DISODS ${ }^{1}$.

\begin{tabular}{|c|c|c|c|c|c|}
\hline State & Index & 1-B-FS & & Faired 1-B-FS & \\
\hline & $w$ & & 0.001 & 0.5 & 4 \\
\hline & Diff & 0 & $1.24 \%$ & $1.21 \%$ & $0.689 \%$ \\
\hline$x$ & & 1.77 & 0.0843 & 0.0899 & 0.277 \\
\hline$\theta_{1}$ & Steady-state error & $58.8 \times 10^{-9}$ & $34.6 \times 10^{-9}$ & $37.2 \times 10^{-9}$ & $56.0 \times 10^{-9}$ \\
\hline$\underline{\theta_{2}}$ & & $26.2 \times 10^{-9}$ & $50.0 \times 10^{-9}$ & $16.9 \times 10^{-9}$ & $25.5 \times 10^{-9}$ \\
\hline$x$ & & 2.01 & 0.228 & 0.225 & 0.495 \\
\hline$\theta_{1}$ & Maximum overshoot & 0.219 & 0.115 & 0.113 & 0.118 \\
\hline$\theta_{2}$ & & 0.149 & 0.0566 & 0.0560 & 0.0644 \\
\hline$x$ & & 4.04 & 3.25 & 3.24 & 3.59 \\
\hline$\theta_{1}$ & Settling time & 1.68 & 0.825 & 0.818 & 0.846 \\
\hline$\theta_{2}$ & & 1.68 & 0.431 & 0.395 & 0.796 \\
\hline & $u^{2} \mathrm{~d} t$ & 15.2 & 5.52 & 5.23 & 6.84 \\
\hline
\end{tabular}

TABLE 5: Performance index of control systems by 2-B-FS, faired 2-B-FSs with I/O data DISODS ${ }^{1}$.

\begin{tabular}{|c|c|c|c|c|c|}
\hline \multirow[t]{3}{*}{ State } & \multirow{2}{*}{$\begin{array}{c}\text { Index } \\
w\end{array}$} & \multirow[t]{2}{*}{ 2-B-FS } & \multicolumn{3}{|c|}{ Faired 2-B-FS } \\
\hline & & & 0.4 & 1.5 & 11 \\
\hline & Diff & $0.213 \times 10^{-3} \%$ & $8.63 \%$ & $1.35 \%$ & $0.146 \%$ \\
\hline$x$ & \multirow{3}{*}{ Steady-state error } & 1.71 & 0.0967 & 0.110 & 0.447 \\
\hline$\theta_{1}$ & & $62.8 \times 10^{-9}$ & $50.7 \times 10^{-9}$ & $44.0 \times 10^{-9}$ & $53.4 \times 10^{-9}$ \\
\hline$\theta_{2}$ & & $30.1 \times 10^{-9}$ & $19.4 \times 10^{-9}$ & $17.1 \times 10^{-9}$ & $24.6 \times 10^{-9}$ \\
\hline$x$ & \multirow{3}{*}{ Maximum overshoot } & 1.94 & 0.268 & 0.322 & 0.664 \\
\hline$\theta_{1}$ & & 0.206 & 0.157 & 0.1166 & 0.1173 \\
\hline$\theta_{2}$ & & 0.143 & 0.0735 & 0.0570 & 0.0695 \\
\hline$x$ & \multirow{3}{*}{ Settling time } & 4.00 & 3.30 & 3.45 & 3.66 \\
\hline$\theta_{1}$ & & 1.62 & 1.46 & 0.827 & 0.881 \\
\hline$\theta_{2}$ & & 1.60 & 0.806 & 0.682 & 0.866 \\
\hline & $u^{2} \mathrm{~d} t$ & 13.9 & 13.2 & 6.24 & 6.64 \\
\hline
\end{tabular}

The control performance of control systems in terms of different weights is shown in Tables 2, 3, 4, and 5, where the control performance includes dynamical performance such as maximum overshoot and settling time, and steady performance such as steady-state error; $T=100 \mathrm{~s}$; diff $=$ $\left(\sum\left(F\left(\mathbf{x}_{s}\right)-v_{s}\right)^{2} / \sum\left(v_{s}\right)^{2}\right) \times 100 \%$ is defined as the relatively adjustment between fuzzy system and its corresponding $\mathrm{I} / \mathrm{O}$ data; the setting time is defined as the time required for the system to settle within $5 \%$ of the steady value, and $\int u^{2} \mathrm{~d} t$ shows the consumption of energy. In particular, the results shown in Tables 4 and 5 are the average result of 100 independent runs. The following results can be seen from the above tables.

(1) The smaller (larger) the weight is, the larger (smaller) the relatively adjustment between the fuzzy system and its corresponding I/O data is (seen from Tables 2, 3,4 and 5). This agrees with our analysis in Section 3.3.

(2) For I/O data DISODS, only small adjustment can make the faired B-FCs have good control effect (seen from Tables 2 and 3). Since the variance of Gaussian white noise of DISODS ${ }^{1}$ is a small value, $(0.001)^{2}$, the good control effect is also available by small adjustment (seen from Tables 4 and 5).

(3) For the relatively exact I/O data DISODS, we can see from Tables 2 and 3 that, almost all the performance index of control systems by faired B-FCs are slightly better than those by B-FCs. Moreover, for faired 1-B-FCs (faired 2-B-FCs), we should note that the energy consumption gets higher (lower) as the weight increasing.

(4) For I/O data DISODS ${ }^{1}$, the control performance of control systems with faired B-FCs is much better than that of control systems with B-FCs (seen from Tables 4 and 5), only except the steady-state error of $\theta_{1}$ of $w=0.001$ in Table 4. Especially, we point out that the energy consumption of faired B-FCs is less than that of the B-FCs.

(5) From Tables 4 and 5, we also found that, for I/O data DISODS ${ }^{1}$, neither faired B-FCs with larger weights 

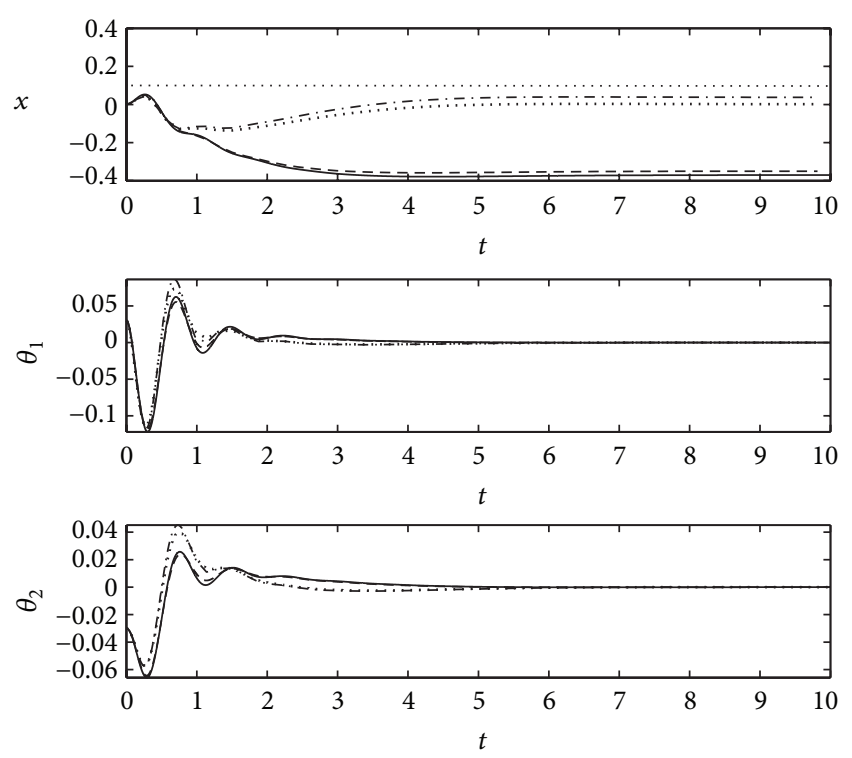

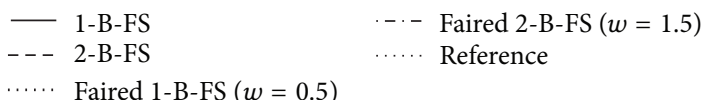

FIGURE 3: Response of controllers by 1-B-FS, 2-B-FS, faired 1-B-FS, and faired 2-B-FS with I/O data DISODS ${ }^{1}$.

nor those with smaller ones can stabilize the double inverted pendulum (figures not shown). In Table 4, when the weight is 0.5 , we can obtain almost the best control performance, especially the energy consumption is the least, while the weight is 1.5 in Table 5 for the same goal. Therefore, one can conclude that the larger (smaller) weights lead to smaller (larger) adjustment to the inexact I/O data DISODS ${ }^{1}$, and both cases are not suitable for the faired B-FSs which are used to construct controllers.

In summary, when the $\mathrm{I} / \mathrm{O}$ data for fuzzy system is relatively exact, the control effect of the faired B-FCs is slightly better than that of the B-FCs, which means the faired B-FSs for the faired B-FCs improve the B-FSs for the B-FCs slightly. While the I/O data for fuzzy system is inexact, the control effect of the faired B-FCs outperforms that of the B-FCs, in this case, the corresponding faired B-FSs reduce adverse effects of the inexact $\mathrm{I} / \mathrm{O}$ data on the corresponding B-FSs as well as improve them significantly.

Remark 4. (1) To compare the control effect of faired B-FCs and B-FCs with inexact I/O data, the variance of the Gaussian white noise added to DISODS should be as small as $(0.001)^{2}$. Otherwise, the corresponding B-FCs cannot stabilize the double invert pendulum. Actually, when the variance of Gaussian white noise is as large as $(0.1)^{2}$, the corresponding faired B-FCs can also stabilize the double invert pendulum.
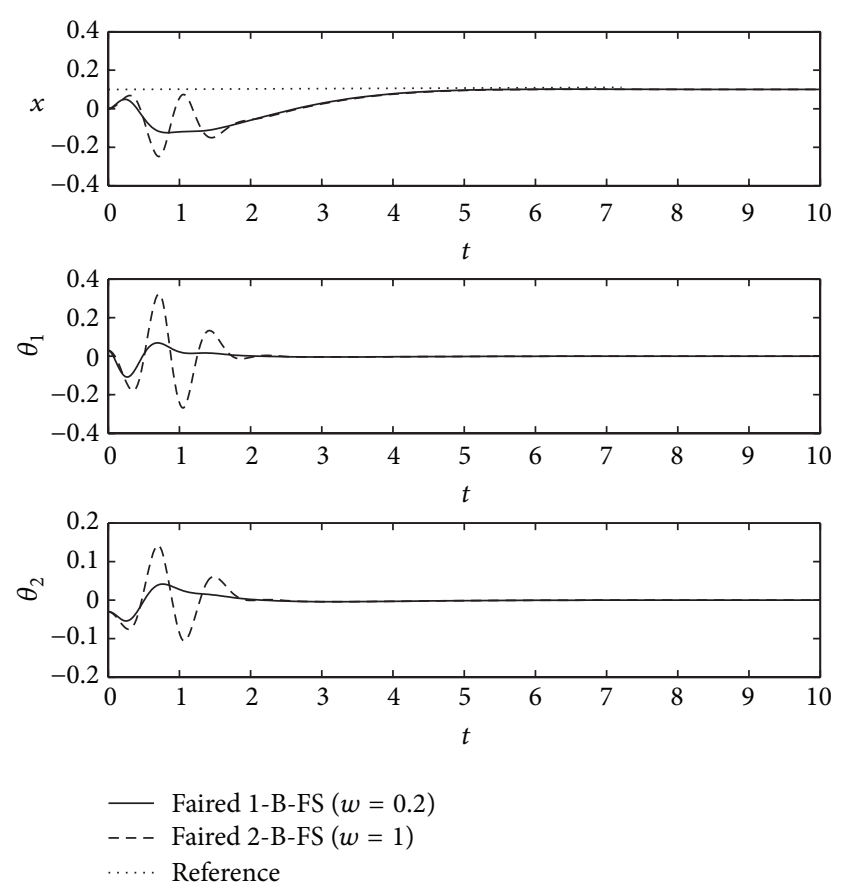

Figure 4: Response of controllers by faired 1-B-FS and faired 2-B-FS with $\mathrm{I} / \mathrm{O}$ data DISODS ${ }^{2}$.

(2) In order to investigate the control capability of the faired B-FCs, we choose

$$
\begin{array}{ccccccc}
\left(z_{i j}^{2}\right)_{7 \times 7}= & \\
\left(\begin{array}{ccccccc}
-0.8333 & -0.8333 & -0.6333 & -0.5 & -0.3333 & -0.1667 & 0 \\
-0.8333 & 0 & 0 & 0 & 0 & 0 & 0.1667 \\
-0.6333 & 0 & 0 & 0 & 0 & 0 & 0.3333 \\
-0.5 & 0 & 0 & 0 & 0 & 0 & 0.5 \\
-0.3333 & 0 & 0 & 0 & 0 & 0 & 0.6333 \\
-0.1667 & 0 & 0 & 0 & 0 & 0 & 0.8333 \\
0 & 0.1667 & 0.3333 & 0.5 & 0.6333 & 0.8333 & 0.8333
\end{array}\right),
\end{array}
$$

and use DISODS ${ }^{2} \triangleq\left\{\left(x_{i}, y_{j}, z_{i j}^{2}\right) \mid i=1,2, \ldots, 7, j=\right.$ $1,2, \ldots, 7\}$ denote the I/O data. In this case, the faired B-FCs can stabilize the double pendulum as well as locate the cart (Figure 4). However, the B-FCs cannot do these.

\section{Conclusion}

In this paper, the energy method in CAGD was utilized to design the faired MISO B-FSs. Based on our generalized approach, the construction of a faired MISO B-FS is equivalent to solve an optimization problem with a strictly convex quadratic objective function. By taking the unique optimal solution vector as the linear combination coefficients of a certain B-FS, a faired MISO B-FS is obtained. For the faired MISO B-FSs, the fairness and difference can be adjusted by modifying the weights in the objective function. This gives us the opportunity to improve the performance of fuzzy systems and fuzzy controllers. Moreover, we use the obtained faired MISO B-FSs to stabilize the double inverted pendulum by modifying the weights. It is concluded that the faired B-FCs 
outperform the B-FCs in the case of exact and inexact $\mathrm{I} / \mathrm{O}$ data. In fact, there are many fairing methods in CAGD. We only choose the energy approach. Moreover, the faired MISO B-FSs are fuzzy systems with robustness. In the future, we will try to fair the B-FSs by other fairing methods and investigate their robustness.

\section{Acknowledgments}

This paper is supported by the National Natural ScienceFoundation of China (no. 61074044, no. 61104038, no. 60834004), and the National 973 Basic Research Program of China (no. 2009CB320602).

\section{References}

[1] M. Biglarbegian, W. Melek, and J. Mendel, "On the robustness of type-1 and interval type-2 fuzzy logic systems in modeling," Information Sciences, vol. 181, no. 7, pp. 1325-1347, 2011.

[2] W. Y. Song, D. G. Wang, and W. G. Wang, "Analysis for perturbation of fuzzy systems modeling," ICIC Express Letters, vol. 3, no. 3, pp. 573-578, 2009.

[3] M. Brown and C. J. Harris, "A nonlinear adaptive controller: a comparison between fuzzy logic control and neurocontrol," IMA Journal of Mathematical Control and Information, vol. 8, no. 3, pp. 239-265, 1991.

[4] J. Zhang and A. Knoll, "Constructing fuzzy controllers with B-spline models: principles and applications," International Journal of Intelligent Systems, vol. 13, no. 2-3, pp. 257-285, 1998.

[5] J. Zhang, K. Van Le, and A. Knoll, "Unsupervised learning of control surfaces based on B-spline models," in Proceedings of the 6th IEEE International Conference on Fussy Systems, pp. 17251730, July 1997.

[6] X. J. Zeng and M. G. Singh, "Approximation accuracy analysis of fuzzy systems as function approximators," IEEE Transactions on Fuzzy Systems, vol. 4, no. 1, pp. 44-63, 1996.

[7] A. Mencattini, M. Salmeri, and A. Salsano, "Sufficient conditions to impose derivative constraints on MISO Takagi-Sugeno fuzzy logic systems," IEEE Transactions on Fuzzy Systems, vol. 13, no. 4, pp. 454-467, 2005.

[8] Q. Luo, W. Yang, and D. Yi, "Kernel shapes of fuzzy sets in fuzzy systems for function approximation," Information Sciences, vol. 178, no. 3, pp. 836-857, 2008.

[9] Z. Long, X. Liang, and L. Yang, "Some approximation properties of adaptive fuzzy systems with variable universe of discourse," Information Sciences, vol. 180, no. 16, pp. 2991-3005, 2010.

[10] L. Hongxing, "Interpolation mechanism of fuzzy control," Science in China E, vol. 41, no. 3, pp. 312-320, 1998.

[11] L. L. Schumaker, Spline Functions: Basic Theory, Cambridge University Press, New York, NY, USA, 2007.

[12] T. Yanhua, L. Hongxing, and X. Jixiang, "Two classes of $B$-spline fuzzy systems and their applications," Journal of Control Theory and Applications, vol. 28, no. 11, pp. 1651-1657, 2011 (Chinese).

[13] T. Yanhua and L. Hongxing, “The MISO B-spline fuzzy systems and its applications in double inverted pendulum".

[14] G. Farin, Curves and Surfaces For CAGD, Morgan Kaufmann Publishers, San Francisco, Calif, USA, 5th edition, 2001.

[15] L.X. Wang, "Stable adaptive fuzzy control of nonlinear systems," IEEE Transactions on Fuzzy Systems, vol. 1, no. 2, pp. 146-155, 1993.
[16] S. Tong and Y. Li, "Observer-based fuzzy adaptive control for strict-feedback nonlinear systems," Fuzzy Sets and Systems, vol. 160, no. 12, pp. 1749-1764, 2009.

[17] T. Wang, S. Tong, and Y. Li, "Robust adaptive fuzzy control for nonlinear system with dynamic uncertainties based on backstepping," International Journal of Innovative Computing, Information and Control, vol. 5, no. 9, pp. 2675-2688, 2009.

[18] S. Tong, C. Liu, and Y. Li, "Fuzzy-adaptive decentralized output-feedback control for large-scale nonlinear systems with dynamical uncertainties," IEEE Transactions on Fuzzy Systems, vol. 18, no. 5, pp. 845-861, 2010.

[19] S. C. Tong, Y. M. Li, G. Feng, and T. S. Li, "Observer-based adaptive fuzzy backstepping dynamic surface control for a class of MIMO nonlinear systems," IEEE Transactions on Systems, Man, and Cybernetics, Part B: Cybernetics, vol. 41, no. 4, pp. 1124-1135, 2011.

[20] Li Hongxing, "To see the success of fuzzy logic from mathematical essence of fuzzy control-on the paradoxical success of fuzzy logic," Fuzzy Systems and Mathematics, vol. 9, no. 4, pp. 1-14, 1995 (Chinese).

[21] H.-X. Li, Z.-H. Miao, and E. S. Lee, "Variable universe stable adaptive fuzzy control of a nonlinear system," Computers \& Mathematics with Applications, vol. 44, no. 5-6, pp. 799-815, 2002.

[22] H. Li, "Adaptive fuzzy controllers based on variable universe," Science in China E, vol. 42, no. 1, pp. 10-20, 1999.

[23] L. Hongxing, M. Zhihong, and W. Jiayin, "Variable universe adaptive fuzzy control on the quadruple inverted pendulum," Science in China E, vol. 45, no. 2, pp. 213-224, 2002.

[24] L. Piegl and W. Tiller, The NURBS Book, Springer, New York, NY, USA, 1996.

[25] X. Shu-fang, The Theory and Technique in Matrix Computation, Peking University Press, Beijing, China, 1995 (Chinese).

[26] Z. Xin-Xiong, Curve and Surface Modeling Technology, Science Press, Beijing, China, 2000 (Chinese).

[27] W. Zai-wen, Least squares method and its application [M.S. thesis], Chinese Academy of Sciences, Academy of Mathematics and Systems Science of Chinese Academy of Sciences, 2004. 


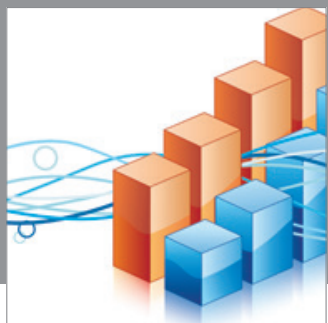

Advances in

Operations Research

mansans

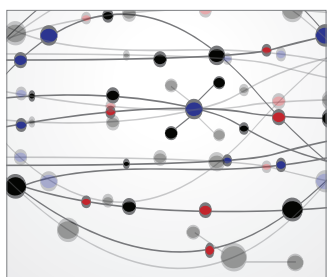

The Scientific World Journal
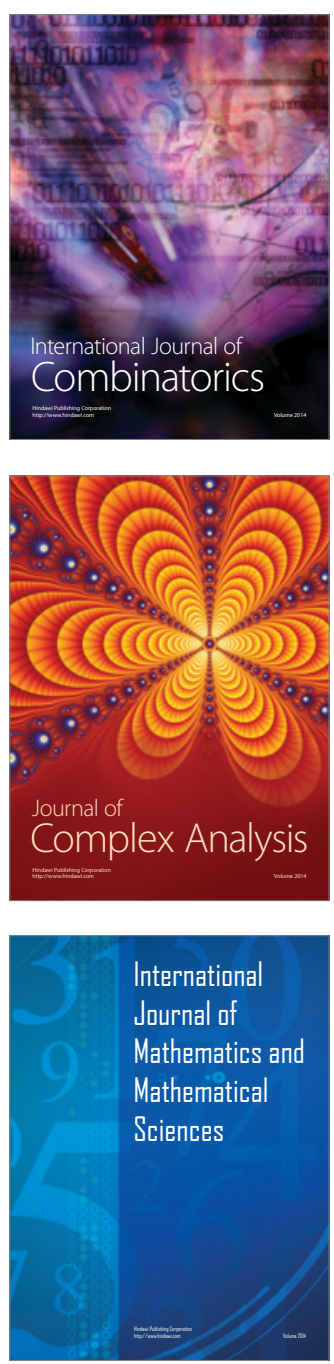
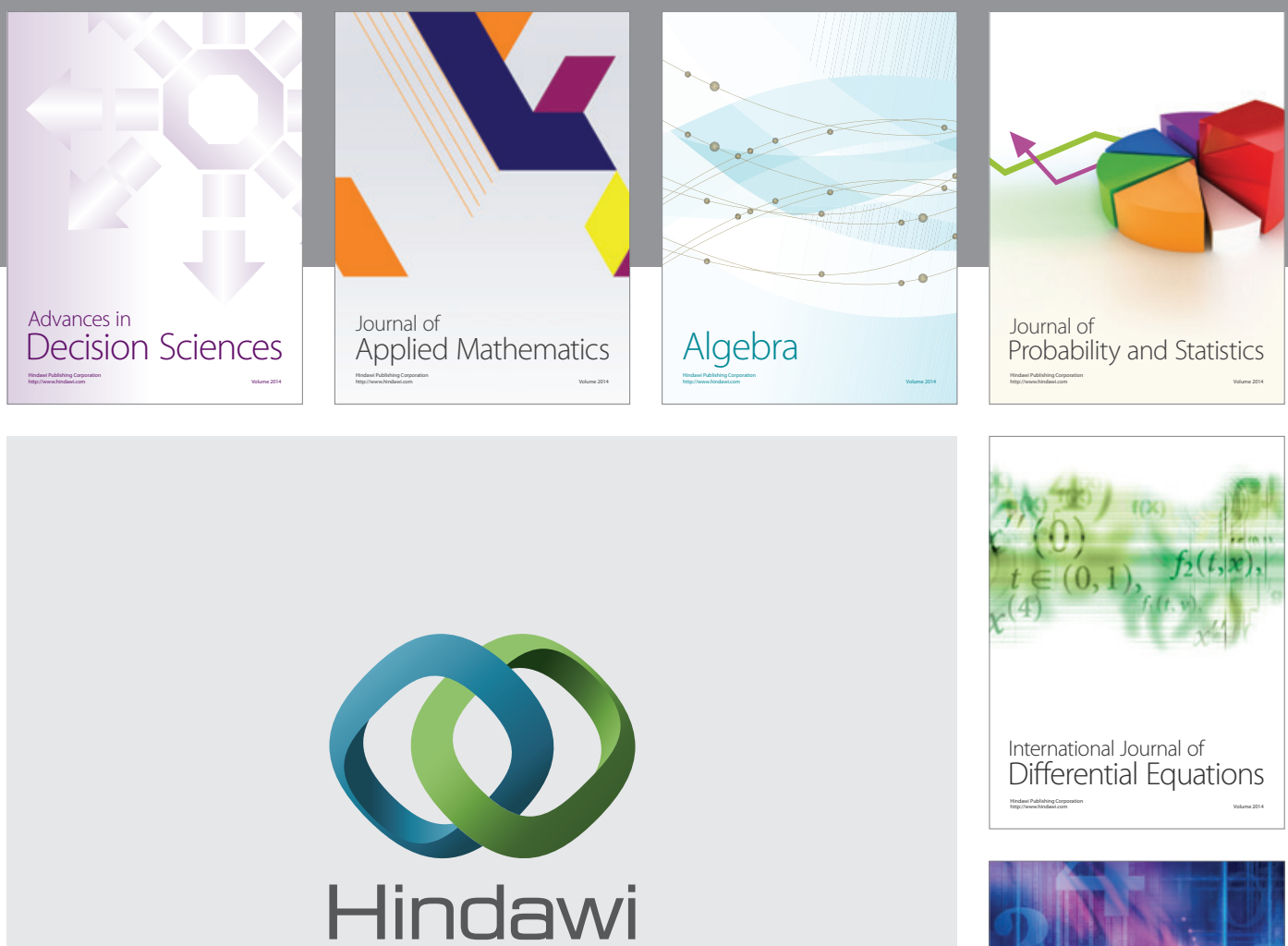

Submit your manuscripts at http://www.hindawi.com
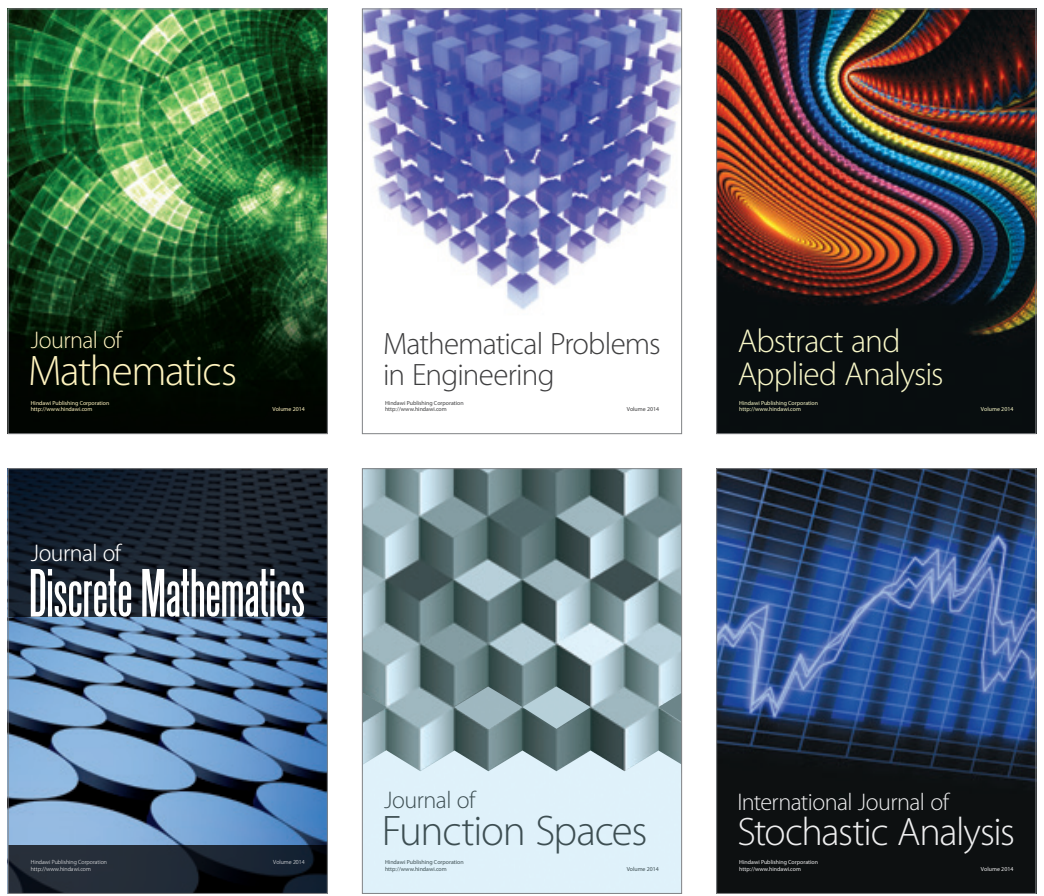

Journal of

Function Spaces

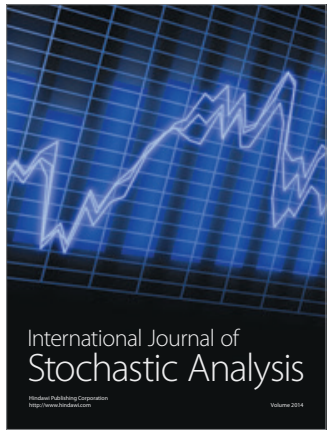

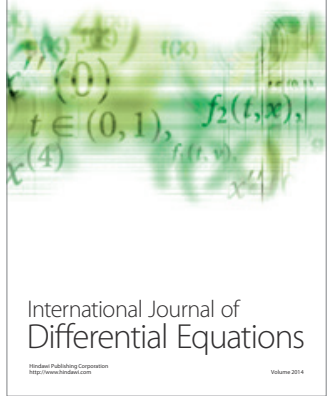
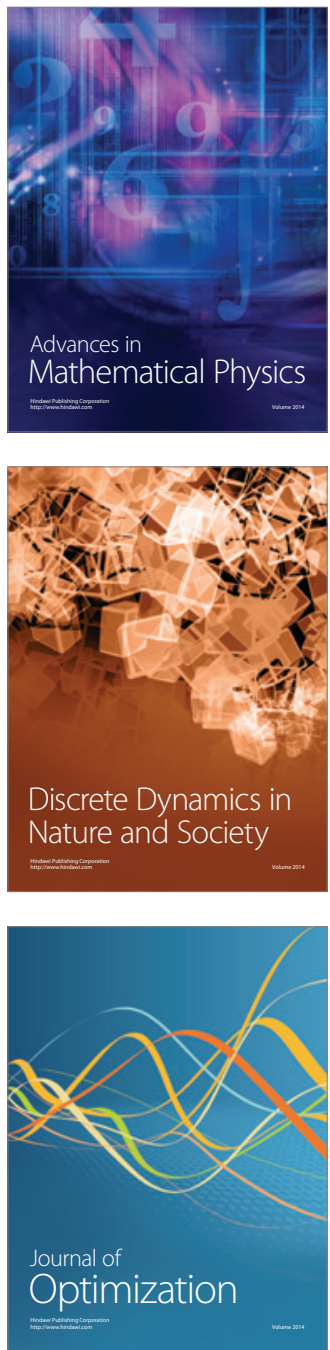